\title{
Coherently Coupled Photonic-Crystal Surface-Emitting Laser Array
}

\author{
Richard J. E. Taylor, David T. D. Childs, Pavlo Ivanov, Ben J. Stevens, Nasser Babazadeh, Jayanta Sarma, \\ Salam Khamas, Alex J. Crombie, Guangrui Li, Gary Ternent, Stephen Thoms, \\ Haiping Zhou, and Richard A. Hogg
}

\begin{abstract}
The realization of a $1 \times 2$ coherently coupled photonic crystal surface emitting laser array is reported. New routes to power scaling are discussed and the electronic control of coherence is demonstrated.
\end{abstract} SEL).

Index Terms-Photonic-crystal surface-emtting laser array (PC-

\section{INTRODUCTION}

$\mathbf{T}$ HERE has been considerable recent interest in photonic crystal surface emitting lasers (PCSELs) due to the demonstration of vertical single mode emission over a large area [1]-[5]. These devices have been shown to give high power that scales with area, low divergence [3], [4], control of beam shape and polarisation [6], [7], and beam steering [8]. Realisation of PCSEL's is normally through wafer fusion [9] or by epitaxial regrowth of voids [1]. There has also been considerable work on all-semiconductor PCSELs whereby expitaxial regrowth is used to fully fill etched holes and leave a photonic crystal PC which is all-semiconductor [10]-[12].

Williams et al. demonstrated single mode emission from an all semiconductor PCSEL [10], and showed a higher mode overlap with the PC region than their void containing counterparts [11]. Fig. 1(a) shows a schematic diagram of the PCSELs considered in this paper where the photonic crystal and active layers are located between $\mathrm{p}$ - and $\mathrm{n}$ - cladding layers, providing in-plane optical wave-guiding. A schematic of a photonic crystal unit cell is shown in Fig. 1(b), where we define A to be the atom shape and material, with $\mathrm{B}$ the background material, $\mathrm{r}$ is atom radius and a is photonic crystal period. Fig. 1(c) shows a schematic of the layer structure of the PCSEL considered in this paper. There

Manuscript received February 1, 2015; revised March 22, 2015; accepted March 26, 2015. This work was supported by the EPSRC under Grant EP/K023195/1. The work of R. J. E. Taylor was supported by the EPSRC Doctoral Prize Fellowship. The Ph.D. work of R. J. E. Taylor and A. J. Crombie was supported by the EPSRC.

R. J. E. Taylor, D. T. D. Childs, P. Ivanov, B. J. Stevens, N. Babazadeh, S. Khamas, A. J. Crombie, G. Li, and R. A. Hogg are with the University of Sheffield, Sheffield S10 2TN, U.K. (e-mail: RJETaylor1@ sheffield. ac.uk; david.childs@sheffield.ac.uk; p.ivanov@sheffield.ac.uk; b.stevens@ sheffield.ac.uk; n.babazadeh@sheffield.ac.uk; s.khamas@sheffield.ac.uk; a.crombie@sheffield.ac.uk; gli13@sheffield.ac.uk; r.hogg@sheffield.ac.uk).

J. Sarma, retired, was with the University of Bath, Bath BA2 7AY, U.K. (e-mail: jsarmabath@gmail.com).

G. Ternent, S. Thoms, and H. Zhou are with the University of Glasgow, Glasgow G12 8QQ, U.K. (e-mail: Gary.Ternent@glasgow.ac.uk; Stephen. Thoms@glasgow.ac.uk; Haiping.Zhou@glasgow.ac.uk).

Color versions of one or more of the figures in this paper are available online at http://ieeexplore.ieee.org.

Digital Object Identifier 10.1109/JSTQE.2015.2417998

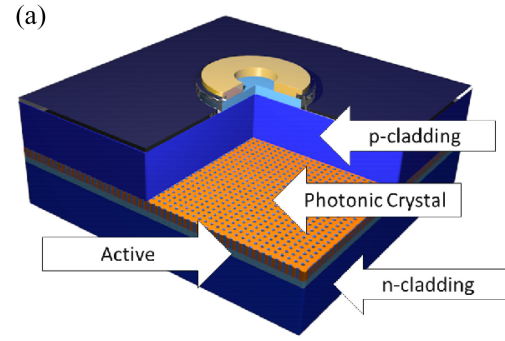

(b)

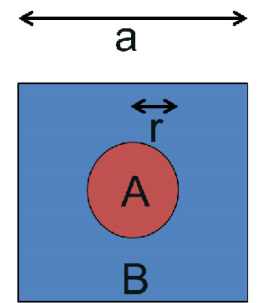

(c)

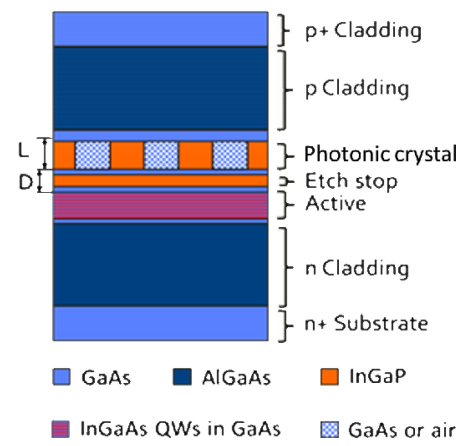

Fig. 1. (a) A schematic of the PCSELs considered in this paper. (b) Schematic of a photonic crystal unit cell. (c) Schematic of device structure.

has been considerable effort to model the effect of atom shape on the performance of the PCSEL, where the engineering of the in-plane electric field allows control of the far-field power, beam shape and polarization [13]. PCSEL arrays have demonstrated powers as high as $35 \mathrm{~W}$ [14] but the devices in these arrays have not demonstrated coherence or that coherence can be controlled.

In this paper we demonstrate that we are able to realize an array of 4 all-semiconductor PCSELs which may be operated $\mathrm{CW}$ with $<1^{\circ}$ divergence (diffraction limited), and essentially identical threshold current densities and emission wavelength. Developments in fabrication and regrowth technologies have allowed a significant improvement over our previous work [10], [12].

We then go on to realise a $1 \times 2$ coherently coupled PCSEL array, controlled by contacted connecting waveguides ("couplers"). These couplers can be operated in loss, transparency or 
gain through a small change in the injected current. We show new opportunities in power scaling through the coupling of adjacent PCSELs and evidence for injection locking between the PCSELs. Finally we demonstrate the electronic control of coherence between PCSEL elements of the array by changing the current applied to the coupler sections. In addition to demonstrating that the array elements are coherent with each other, we demonstrate that this shows for the first time that the individual PCSELs emit coherently across their surface.

\section{PCSEL FABRICATION AND REALISATION}

In this section we describe device growth and fabrication and show the characteristics of individual PCSELs. Initial growth occurred on a GaAs substrate, $3^{\circ}$ off toward (110), consisting of: $1.5 \mu$ m of $\mathrm{n}-\mathrm{Al}_{0.4} \mathrm{Ga}_{0.6}$ As, three $8 \mathrm{~nm} \mathrm{In}_{0.2} \mathrm{Ga}_{0.8}$ As quantum wells with $20 \mathrm{~nm}$ GaAs barriers, a $40 \mathrm{~nm} \mathrm{p}-\mathrm{In}_{0.48} \mathrm{Ga}_{0.52} \mathrm{P}$ etch stop layer and a $20 \mathrm{~nm} \mathrm{p}-\mathrm{GaAs}$ buffer layer. Above this is was a $150 \mathrm{~nm}$ of $\mathrm{p}-\mathrm{In}_{0.48} \mathrm{Ga}_{0.52} \mathrm{P}$ and a $20 \mathrm{~nm}$ p-GaAs terminating layer. Electron beam lithography and reactive ion etching was utilised to transfer a square lattice, circular dielectric cylinder photonic crystal pattern into a $\mathrm{SiO}_{2}$ hard mask. This pattern was transferred into the InGaP layer by an $\mathrm{CH}_{4} / \mathrm{H}_{2} / \mathrm{O}_{2}$ reactive ion etch. The hard mask was then removed and regrowth to fill the PC structure, planarise the waveguide, and grow upper p-doped cladding layers was undertaken in an MOVPE reactor. Prior to device fabrication, the MOVPE process was optimized due to the high aspect ratio of the PC features [10]-[12].

The regrowth procedure involves the patterened wafer being etched in buffered HF for $30 \mathrm{~s}$ prior to being loaded into the growth reactor. There is a trade off in the choice of regrowth temperature. High temperatures are required to desorb natural oxides, yet result in As:P exchange, which we aim to minimise. Low temperatures lead to the formation of threading dislocations. These constraints on growth temperature remove an element of freedom when trying to ensure good planarization during the regrowth process. In order to optimise the regrowth process, samples identical to the final PCSEL design were realised which differed by having a PC layer $\left(\mathrm{p}-\mathrm{In}_{0.48} \mathrm{Ga}_{0.52} \mathrm{P}\right)$ of $150 \mathrm{~nm}$ in thickness. Following regrowth, devices were formed by etching a $400 \mathrm{~nm}$ deep $100 \mu$ m diameter mesa in the $\mathrm{p}+\mathrm{GaAs}$ contact layer above the centre of the photonic crystal. An annular gold contact was defined, providing a $52 \mu \mathrm{m}$ aperture for light extraction. The electrically driven region (100 $\mu \mathrm{m}$ diameter plus current spreading) is smaller than the regrown photonic crystal area $(150 \mu \mathrm{m} \times 150 \mu \mathrm{m})$.

Fig. 2 shows the CW electroluminescence (EL) spectra of a typical all-semiconductor PCSEL operating at room temperature at $100 \mathrm{~mA}$ showing emission at $991 \mathrm{~nm}$, the inset is zoomed in on the same spectra, showing that the full-width at half-maximum (FWHM) is $0.5 \mathrm{~nm}$.

Fig. 3 shows the CW light output-current (LI) curve of a typical PCSEL operating at room temperature showing the threshold current of the device to be $65 \mathrm{~mA}$, the inset shows the far-field of the same device operating at $100 \mathrm{~mA}$. The FWHM of the emitted beam is $\sim 1.3^{\circ}$ in each direction, in excellent agreement with the diffraction angle of $0.991 \mu \mathrm{m}$ light through a

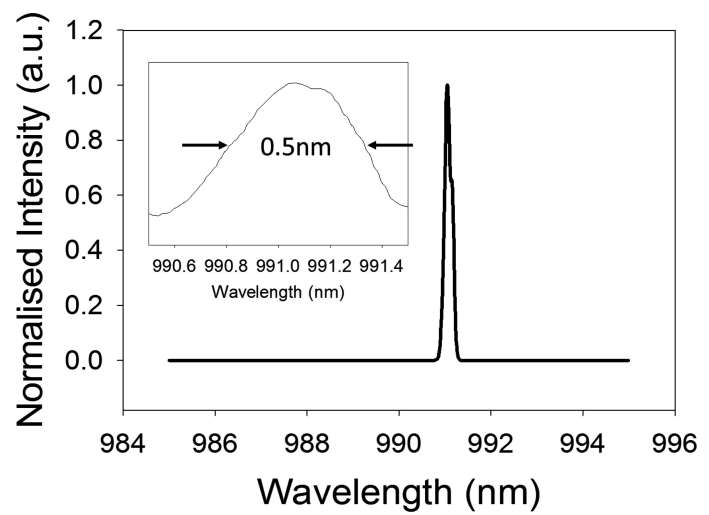

Fig. 2. EL spectra of a typical all-semiconductor PCSEL, inset shows the spectra close to $991 \mathrm{~nm}$.

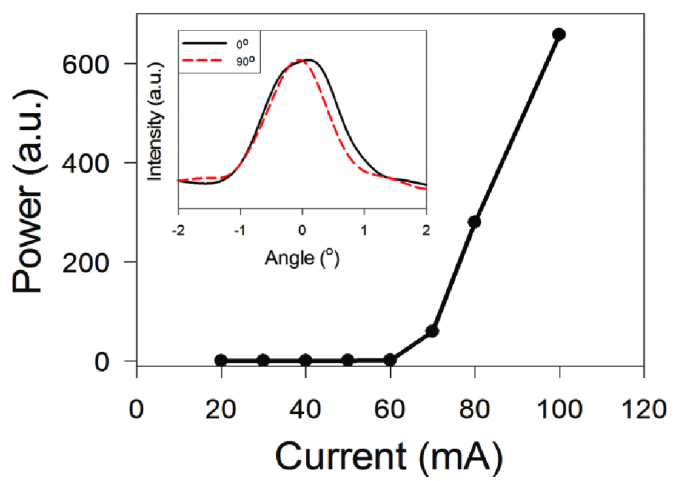

Fig. 3. Light output-current curve of a typical all-semiconductor PCSEL inset shows the far field pattern of the same device operating well above threshold.

$52 \mu \mathrm{m}$ diameter aperture. This shows the first demonstration of an all-semiconductor PCSEL operating continuous wave at room temperature and shows a lower divergence than our previously realized devices.

The threshold current $\left(I_{\mathrm{th}}\right)$ of the device is $65 \mathrm{~mA}$, which gives a threshold current density $\left(J_{\mathrm{th}}\right)$ of $\sim 0.8 \mathrm{kA} / \mathrm{cm}^{2}$. Previously studied all semiconductor PCSELs in Williams et al., had a $J_{\text {th }}$ of $\sim 10 \mathrm{kA} / \mathrm{cm}^{2}$. The output poer at $100 \mathrm{~mA}$ was $\sim 100 \mu \mathrm{W}$, giving a slope efficiency of $3.3 \mu \mathrm{W} / \mathrm{mA}$. This is a significant reduction in the $J_{\text {th }}$ and can be attributed to a number of possible differences between the two devices. Firstly, these devices have an atom radius of $0.4 \mathrm{a}$ (compared to $\sim 0.2$ ) which is shown in Taylor et al. [15] to give a high coupling coefficient, reducing threshold gain. However, this enhanced coupling will also increase out-of-plane scattering that acts as an internal loss prior to lasing. An improvement in sidewall verticality in the present structures is expected to reduce parasitic out-of-plane scattering. Secondly the cleaning process before re-growth was improved, and is likely to give the main enhancement of threshold current density, finally the MOVPE re-growth process has been improved.

\section{COUPLED ARRAY}

In this section we characterise the PCSEL array. The array consists of four PCSELs (as describe in Section I) in a $2 \times 2$ array separated by $1 \mathrm{~mm}$, shown schematically in Fig. 4 . The cou- 


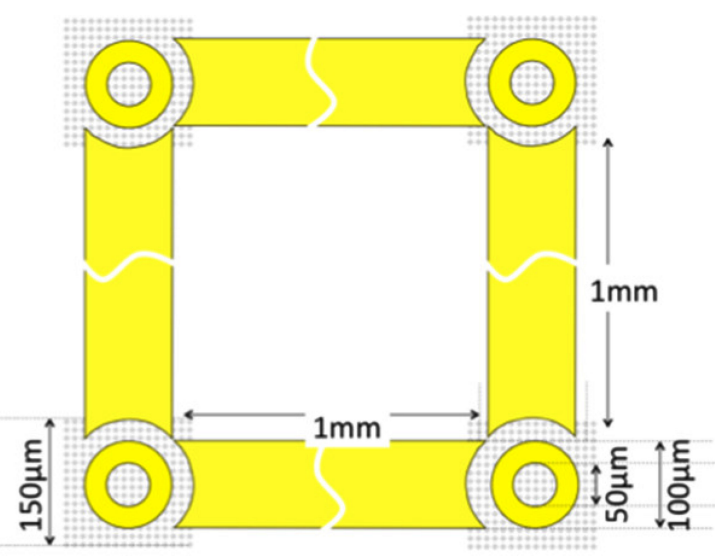

Fig. 4. Schematic of a $1 \times 2$ PCSEL array.

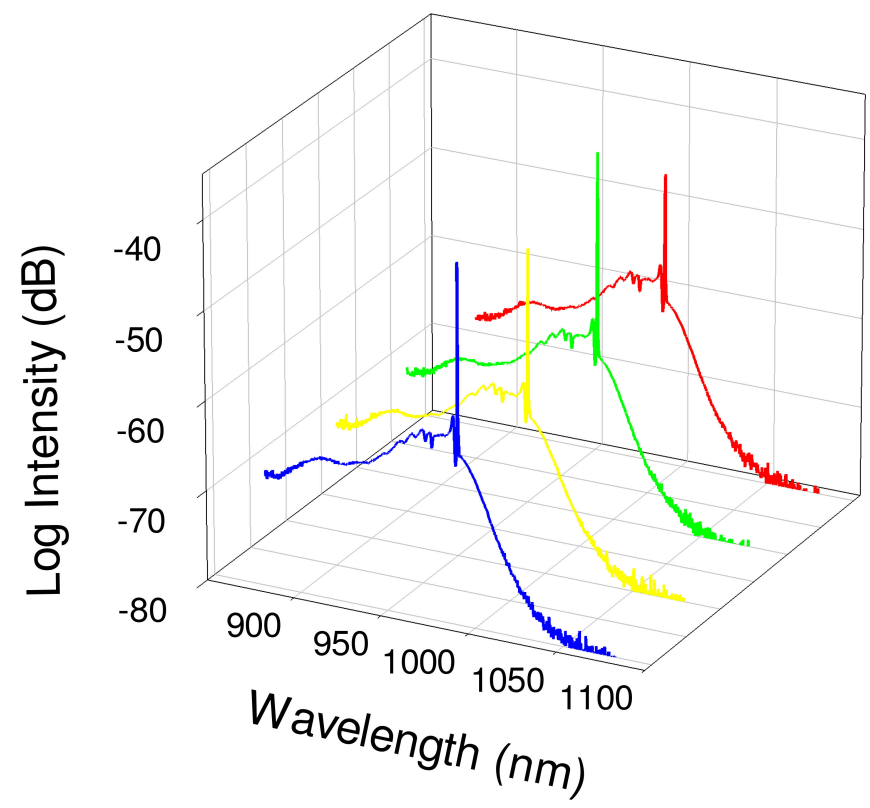

Fig. 5. Spectra of four neighboring devices on the same coupled device with each device operated at $100 \mathrm{~mA} \mathrm{CW}$ at room temperature.

pler region that separate the PCSELs is contacted but contains no photonic crystal, the coupler and the PSCEL are electrically isolated with a resistance of $\sim 2 \mathrm{k} \Omega$ between them. The array takes advantage of the in-plane coupling of the photonic crystal.

Fig. 5 shows the E-L spectra of four PCSELs from the same array, the devices are all operated $\mathrm{CW}$ at room temperature. The peak lasing wavelength of the 4 PCSELs is $991.20 \pm 0.06 \mathrm{~nm}$ and the lasing linewidth of the PCSELs is $0.50 \pm 0.05 \mathrm{~nm}$. Fig. 6 shows the L-I curve of four PCSELs operated continuous wave at room temperature, from the same coupler, the threshold of the four devices is $66 \pm 2 \mathrm{~mA}$. Figs. 5 and 6 demonstrate that within the $2 \times 2$ array the four devices are essentially identical with the difference in the lasing wavelength of each PCSEL being significantly smaller than their lasing linewidths.

In the following we refer to a number of experiments where we refer to the coupler section being in gain or loss. Length characterization of as-cleaved lasers with the same contact method and width as the coupler indicate a transparency current

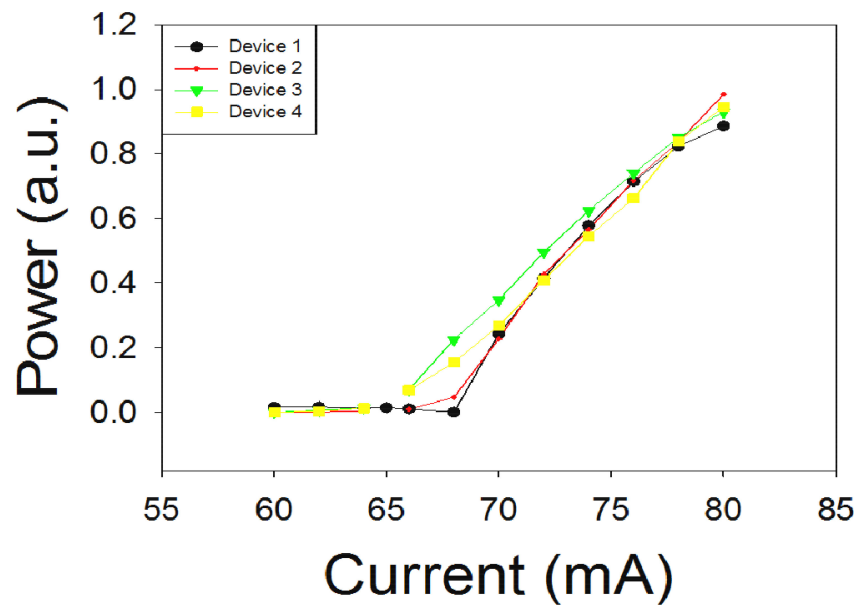

Fig. 6. Light output-current curve of four neighbouring PCSELs from the same $1 \times 2$ array.

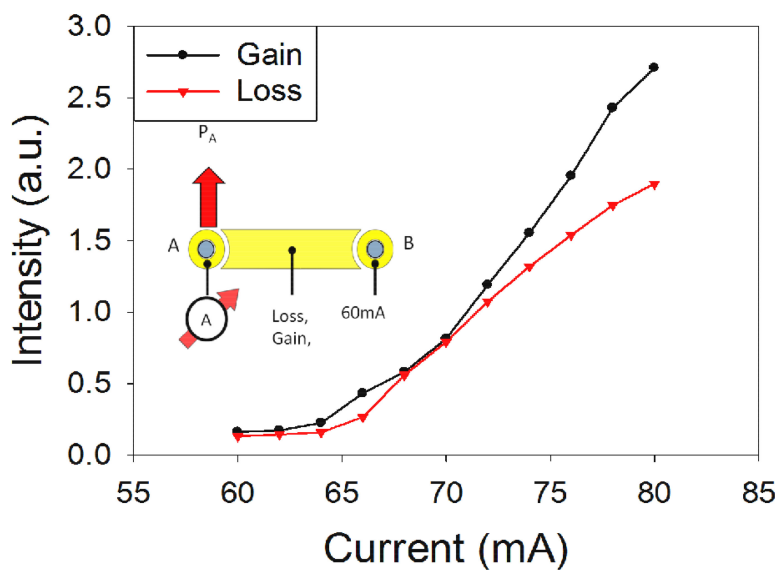

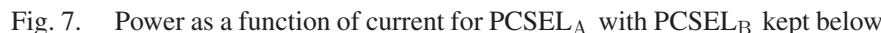
threshold and the coupler in loss (red dashed) and in gain (black solid), inset shows a schematic of the array indicating how experiment was setup.

density of $\sim 220 \pm 4 \mathrm{Acm}^{-2}$. We experimentally determined two current levels at which we observed evidence for coherent coupling and injection locking which we refer to as the coupler being in gain $\left(220 \mathrm{Acm}^{-2}\right)$, and a current level at which we could not observe such effects, which we refer to as the coupler being in loss $\left(200 \mathrm{Acm}^{-2}\right)$.

Fig. 7 shows output power of $\mathrm{PCSEL}_{\mathrm{A}}$ as a function of current for a $1 \times 2$ PCSEL array where PCSEL $_{B}$ is driven sub-threshold $\left(I_{B}=60 \mathrm{~mA}, I_{\text {Thresh }}=68 \mathrm{~mA}\right)$ at, or close to, transparency. The PCSEL ${ }_{\mathrm{A}}$ current is varied from $60 \mathrm{~mA}$ to $80 \mathrm{~mA}$ with the coupler in gain (black) or loss (red). This is shown schematically in the inset. With the coupler in loss the curve shows the lasing threshold of a single PCSEL with a threshold of $65 \mathrm{~mA}$. Reducing the coupler current further did not significantly affect the results. With the coupler in gain the same threshold can be observed but the slope efficiency is increased. We attribute this increase in slope efficiency to light travelling in-plane, exiting PCSEL $_{A}$ being amplified along the coupler, subsequently reflected from PCSEL $_{B}$ once more amplified along the coupler, and subsequently contributing to an increased lasing power 


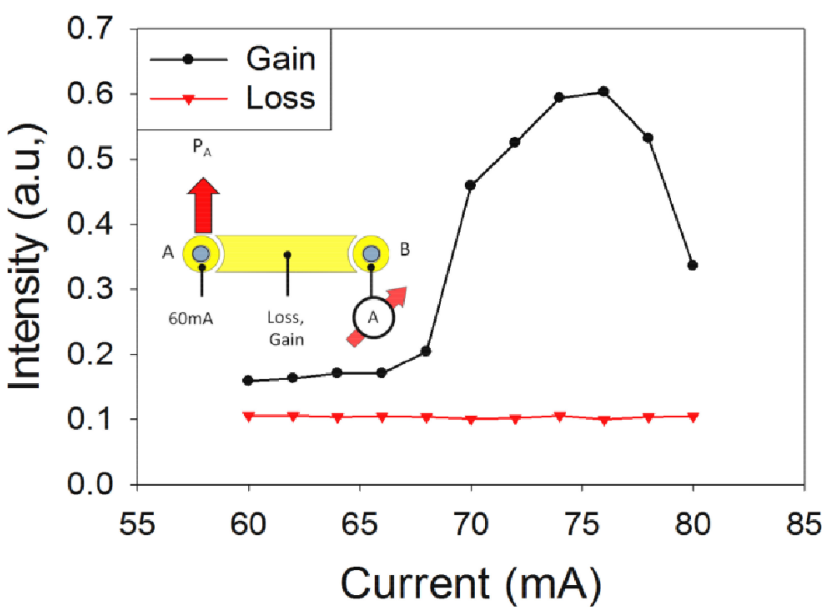

Fig. 8. Power as a function of current for PCSEL A below threshold, PCSEL P $_{B}$ current varying from 60 to $80 \mathrm{~mA}$ and the coupler in loss (red dashed) and in gain (black solid), inset shows a schematic of the array indicating how experiment was setup.

from PCSEL $_{\mathrm{A}}$. Current leaking from the coupler into PCSEL $\mathrm{A}$ may result in a reduction in threshold but cannot explain their increased slope efficiency observed here. Similarly, additional light generated by the coupler being injected into the PCSEL cannot explain the increased slope efficiency.

Fig. 8 shows output power of PCSEL $_{A}$ as a function of current for a $1 \times 2$ PCSEL array where PCSEL A $_{\mathrm{A}}$ is driven sub-threshold $\left(I_{A}=60 \mathrm{~mA}, I_{\text {Thresh }}=65 \mathrm{~mA}\right)$ at, or close to, transparency. The PCSEL $_{B}$ current is varied from 60 to $80 \mathrm{~mA}$ with the coupler in gain (black) or loss (red). This is again shown schematically in the inset. With the coupler in loss, the output power of PCSEL A $_{A}$ is independent of the current applied to $\operatorname{PCSEL}_{\mathrm{B}}$, demonstrating that in this case the two PCSELs are optically, electrically, and thermally isolated. With the coupler in gain the power emitted from the PCSEL $_{A}$ device significantly increases for PCSEL ${ }_{B}$ currents greater than $68 \mathrm{~mA}$ and rolls over for currents greater than $75 \mathrm{~mA}$. The onset of the increase in power occurs at the

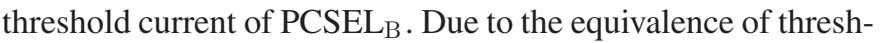
old currents, this behaviour is attributed to waveguided laser emission from PCSEL $_{B}$ being out-coupled by the grating in PCSEL $_{A}$. This increase in emission power from an observed PCSEL offers a route to power scaling. The apparent thermal roll-over is attributed to self heating of $\mathrm{PCSEL}_{\mathrm{B}}$ resulting in detuning of the PCSEL $\mathrm{B}_{\mathrm{B}}$ emission from the scattering wavelength of the grating PCSEL . $_{\text {. }}$

Fig. 9(a) shows the results of EL spectral measurements of the $1 \times 2$ PCSEL array where PCSEL $_{B}$ is lasing $(80 \mathrm{~mA})$, the coupler is in loss and the current in $\mathrm{PCSEL}_{\mathrm{A}}$ is varied from 60 to $80 \mathrm{~mA}$. The inset shows a schematic of this drive condition, similar to that in Fig. 7, but now with PCSEL $_{B}$ lasing. As the two PCSELs are optically isolated (confirmed by reducing the coupler current to even lower levels), these spectra show the spectral behaviour of the un-coupled $\mathrm{PCSEL}_{\mathrm{A}}$. Fig. 9(b) shows the same measurement, but with the coupler in gain. At PCSEL $A$ currents below threshold, the spectra show emission attributed to out-coupling of $\mathrm{PCSEL}_{B}$ emission via the PC in $\mathrm{PCSEL}_{\mathrm{A}}$. A higher intensity of emission is observed when $\mathrm{PCSEL}_{\mathrm{A}}$ reached threshold.
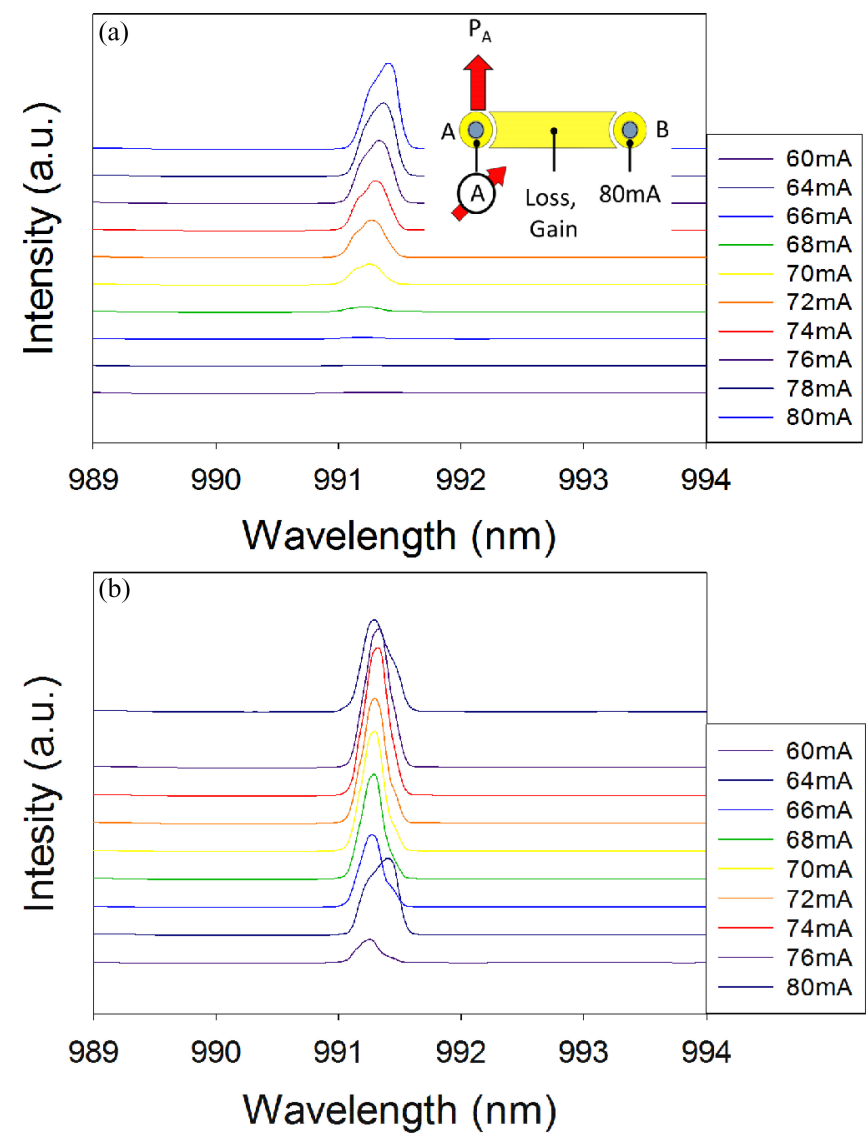

Fig. 9. EL spectra of $1 \times 2$ PCSEL array where the current in PCSEL $_{\mathrm{A}}$ is varied from 60 to $80 \mathrm{~mA}$, the coupler is in loss (a) or gain (b) and PCSEL current is $80 \mathrm{~mA}$.

Fig. 10(a) and (see Fig. 10(b)) plots the peak wavelength as a function of applied current extracted from Fig 9(a) (see Fig. 9(b)). For Fig. 10(a) the peak wavelength monotonously increases from $991.20 \mathrm{~nm}$ to $991.43 \mathrm{~nm}$ over a current range from 66 to $80 \mathrm{~nm}$, giving a peak shift of $0.016 \mathrm{~nm} / \mathrm{mA}$. As we are observing an isolated PCSEL, this shift is attributed to the self-heating of the device. From previous work [5], this indicates $\mathrm{a} \sim 6-7^{\circ} \mathrm{C}$ increase in junction temperature.

While Fig. 10(b) shows the same experiment, but now with the coupler in gain. For the same range of currents applied to PCSEL $_{\mathrm{A}}$ the peak wavelength remains constant at $991.30 \pm$ $0.03 \mathrm{~nm}$. In this case, the self-heating induced shift of the lasing wavelength of $\mathrm{PCSEL}_{\mathrm{A}}$ is not observed. We attribute this to PCSEL $_{A}$ now being injection locked to PCSEL ${ }_{B}[16]$, [17], the injection locking occurs through the transparent coupler.

Fig. 11 shows the EL spectra from PCSEL $_{\mathrm{A}}$ in the same $1 \times 2$ PCSEL array where the current in $\mathrm{PCSEL}_{\mathrm{A}}$ is held constant $80 \mathrm{~mA}$, the coupler is in gain, and the PCSEL $_{\mathrm{B}}$ current is varied from 60 to $80 \mathrm{~mA}$. At currents bellows the lasing threshold of PCSEL $_{B}$ laser emission from PCSEL $_{A}$ is observed. As PCSEL $_{B}$ current is increased, total output power is increased, and at the highest PCSEL $_{B}$ currents a reduction in peak lasing wavelength is observed.

Fig. 12 shows the peak wavelength extracted from Fig. 11. A reduction in $\operatorname{PCSEL}_{\mathrm{A}}$ peak lasing wavelength is observed 


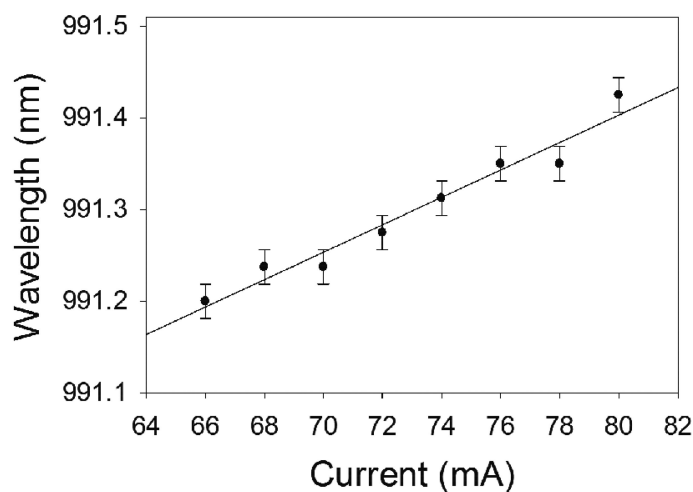

(a)

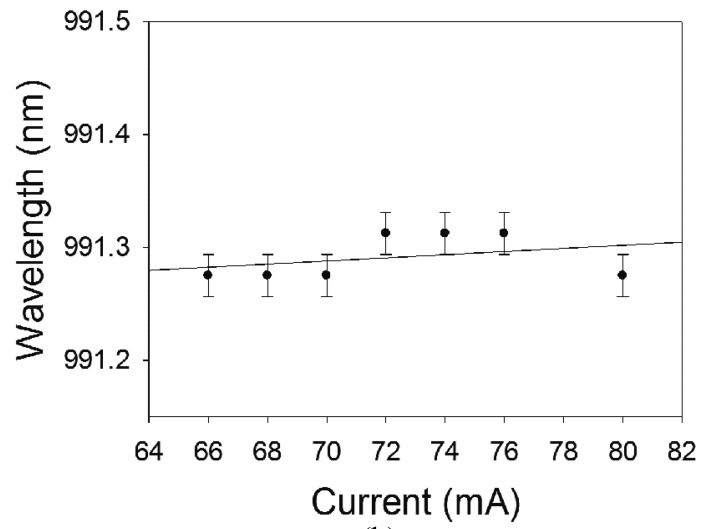

(b)

Fig. 10. Peak wavelength versus applied current for a $1 \times 2$ PCSEL array where the current in $\mathrm{PCSEL}_{\mathrm{A}}$ is varied from 66 to $80 \mathrm{~mA}$, the coupler is in loss (a) or gain (b) and PCSEL $_{\mathrm{B}}$ current is $80 \mathrm{~mA}$.

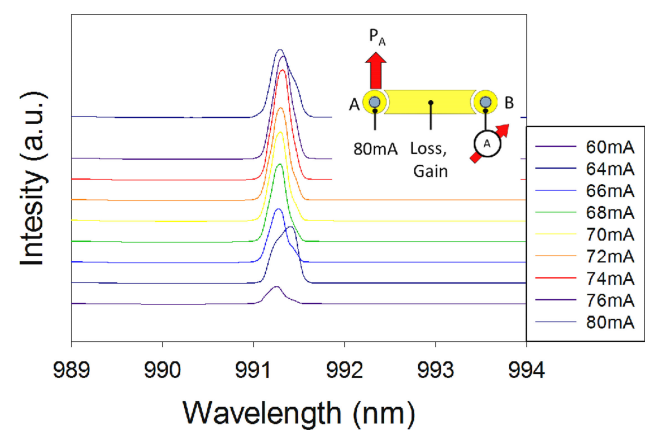

Fig. 11. EL spectra of $1 \times 2$ PCSEL array where the current in $\mathrm{PCSEL}_{\mathrm{A}}$ is $80 \mathrm{~mA}$ and the current in PCSEL $_{\mathrm{B}}$ is varied from 60 to $80 \mathrm{~mA}$, with the coupler in Gain.

when PCSEL $_{\mathrm{B}}$ reaches threshold. This is again attributed to the injection locking of the two PCSELs.

\section{Electronic CONTROL OF COHERENCE}

In this section we use the coupled array to electronically control the coherence of adjacent devices by the control of current applied to the coupler region. In order to ensure that the phase matching condition is met regardless of coupler current, we designed the coupler length to be $1 \mathrm{~mm}$. This gives a Fabry-Pérot (FP) mode spacing of $0.13 \mathrm{~nm}$, meaning that the phase matching

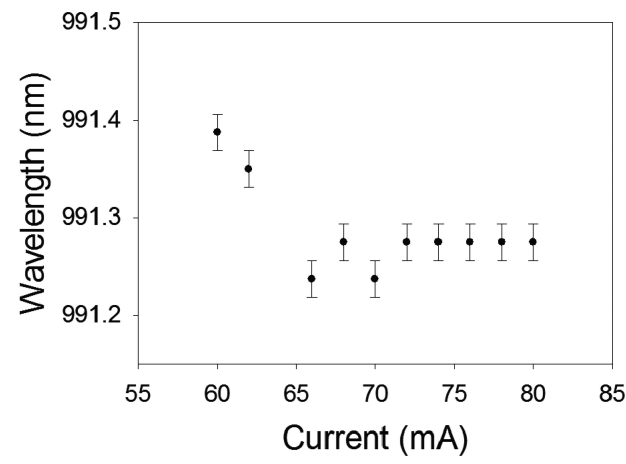

Fig. 12. Peak wavelength versus applied current for a $1 \times 2$ PCSEL array

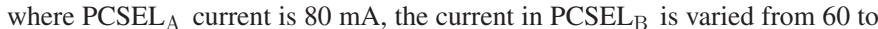
$80 \mathrm{~mA}$ and the coupler is in gain.

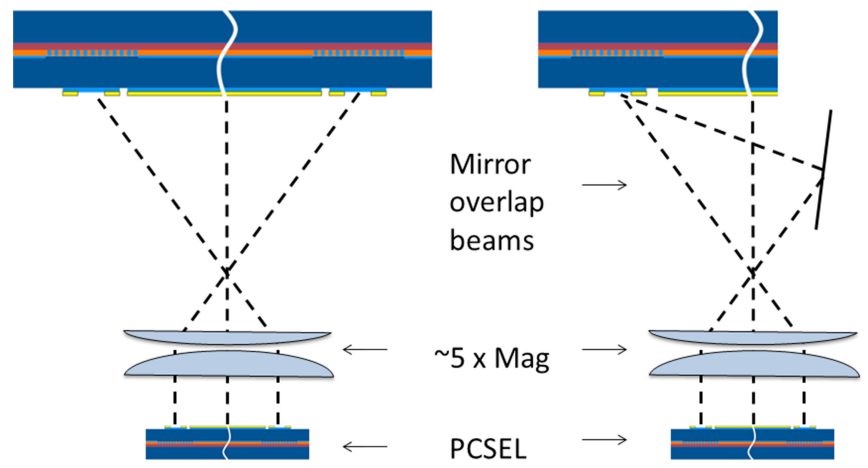

Fig. 13. Schematic of experimental setup for coherence control.

condition is met for a hand-full of F-P modes within the lasing line-width.

Fig. 13 shows a schematic of the experimental setup used to show (or show the absence of) coherence of adjacent PCSELs. The left hand image schematically shows the near field image of a PCSEL array magnified onto a camera. The right hand image shows how the insertion of a mirror reflects the near-field image of one PCSEL so that it overlays the image of its neighbouring PCSEL. The observation of an interference pattern will therefore provide evidence of coherence between the PCSELs.

Fig. 14 shows results from this experiment. The top left image shows the case where both devices are sub-threshold and the coupler is in loss. This shows a low intensity image with no evidence for an interference pattern, demonstrating that the devices are incoherent. The top right image shows the case where both devices are subthreshold and the coupler is in gain. For this case there are still no fringes but the intensity is increased. This is attributed to aditional spontaneous emission from the coupler being out-coupled by the two PCSELs.

The bottom left image shows the case where both devices are lasing $(80 \mathrm{~mA})$ and the coupler is in loss. This shows a further increase in intensity again (due to lasing), but no interference pattern is observed indicating that even though the devices are lasing, they are mutually incoherent. The bottom right image shows the case where both devices are lasing and the coupler is in gain. A clear inteference pattern is observed. The calculated fringe spacing for our experimental set-up is $36 \pm 4 \mu \mathrm{m}$, the 

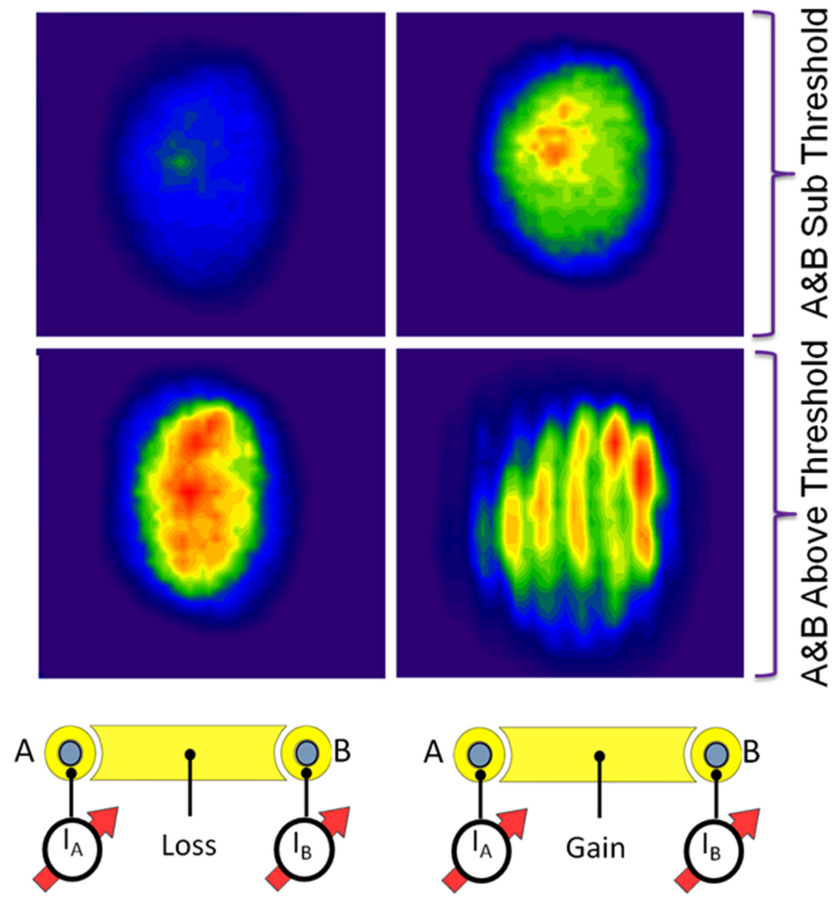

Fig. 14. Near field images of two PCSELs overlaid where both devices are sub threshold and the coupler is in loss (top left) both devices are subthreshold and the coupler is in gain (top right) both devices are lasing and the coupler is in loss (bottom left) and both devices are lasing and the coupler is in gain with schematics showing both where current is applied.

measured spacing $36.6 \pm 5 \mu \mathrm{m}$, which are in excellent agreement. In addition, the observation of such an interference patter demonstrates coherence over the entire surface of the individual PCSELs. This demonstration of mutual coherence of the two PCSELs, allows the realization of the Young's double-slit experiment in the solid state.

\section{SUMMARY}

We have demonstrated a $1 \times 2$ array of all-semiconductor PCSELs which may be operated $\mathrm{CW}$ with $<1^{\circ}$ divergence (diffraction limited), and essentially identical threshold current densities and emission wavelength. We subsequently realised a $1 \times 2$ coherently coupled PCSEL array, controlled by contacted connecting coupler waveguides. We have shown new opportunities in power scaling through the coupling of adjacent PCSELs and shown spectroscopic evidence for injection locking between the PCSELs. Finally, we demonstrated the electronic control of coherence between PCSEL elements of the array by changing the current applied to the coupler sections.

\section{ACKNOWLEDGMENT}

R. J. E. Taylor would like to thank Prof. S. Sweeney and Prof. D. Mowbray for fruitful discussions.

\section{REFERENCES}

[1] K. Hirose et al., "Watt-class high-power, high-beam-quality photoniccrystal lasers," Nature Photon., vol. 8, pp. 406-411, 2014.
[2] E. Miyai et al., "Lasers producing tailored beams," Nature, vol. 441, p. 946, 2006.

[3] M. Imada et al., "Coherent two-dimensional lasing action in surfaceemitting laser with triangular-lattice photonic crystal structure," Appl. Phys. Lett., vol. 75, no. 3, p. 316, 1999.

[4] D. Ohnishi, T. Okano, M. Imada, and S. Noda, "Room temperature continuous wave operation of a surface emitting two dimensional photonic crystal laser," Opt. Exp., vol. 12, no. 8, pp. 1562-1568, 2004.

[5] K. Sakai, E. Miyai, T. D. Ohnishi, T. Okano, and S. Noda, "Lasing bandedge identification for a surface-emitting photonic crystal laser," IEEE J. Sel. Areas Commun., vol. 23, no. 7, pp. 1335-1340, Jul. 2005.

[6] Y. Kurosaka, K. Sakai, E. Miyai, and S. Noda, "Controlling vertical optical confinement in two-dimensional surface-emitting photonic-crystal lasers by shape of air holes," Opt. Exp., vol. 16, no. 22, pp. 1848-18494, Oct. 2008.

[7] S. Noda, M. Yokoyama, A. Chutinan, M. Imada, and M. Mochizuki, "Polarization mode control of two-dimensional photonic crystal laser by unit cell structure design," Science, vol. 293, pp. 1123-1125, 2001.

[8] Y. Kurosaka et al., "On-chip beam-steering photonic-crystal lasers," $N a$ ture Photon., vol. 4, pp. 447-450, 2010.

[9] M. Imada, S. Noda, H. Kobayashi, and G. Saki, "Characterization of a distributed feedback laser with air/semiconductor gratings embedded by the wafer fusion technique," IEEE J. Quantum Electron., vol. 35, no. 9, pp. 1277-1283, Sep. 1999.

[10] D. Williams et al., "Epitaxially regrown GaAs-based photonic crystal surface emitting laser," IEEE Photon. Technol. Lett., vol. 24, no. 11, pp. 966-968, Jun. 2012.

[11] D. Williams et al., "Optimisation of coupling between photonic crystal and active elements in an epitaxially regrown GaAs based photonic crystal surface emitting laser," Jpn. J. Appl. Phys., vol. 51, pp. 02BG05-1-02BG05-3, Feb. 2012.

[12] R. J. E. Taylor et al., "All-semiconductor photonic crystal surface emitting lasers based on epitaxial regrowth," IEEE J. Sel. Topics Quantum Electron., vol. 19, no. 4, p. 4900407, Jul. 2013.

[13] Y. Kurosaka et al., "Band structure observation of 2D photonic crystal with various v shaped air hole arangements," IEICE Electron. Exp., vol. 6, no. 13, pp. 966-971, 2009.

[14] T. Sakaguchi1 et al., "Surface-emitting photonic-crystal laser with 35W peak power," presented at the Conf. Lasers Electro-Opt. Int. Quantum Electron., Baltimore, MD, USA, 2009.

[15] R. J. E. Taylor et al., "Band structure and waveguide modelling of epitaxially regrown photonic crystal surface emitting lasers," J. Phys. D, vol. 46, no. 26, p. 264005 , Jul. 2013.

[16] S. Kobayashi and T. Kimura, "Injection locking in AlGaAs semiconductor laser,' IEEE J. Quantum Electron., vol. QE-17, vol. 17, no. 5, pp. 681-689, May 1981.

[17] R. Lang, "Injection locking properties of semiconductor laser," IEEE J. Quantum Electron., vol. QE-18, no. 6, pp. 976-983, Jun. 1982.

Richard J. E. Taylor received the B.Eng. degree in electrical engineering, the M.Sc. degree in semiconductor photonics, and the Ph.D. degree from the University of Sheffield, Sheffield, U.K., in 2010, 2011, and 2015, respectively. He is currently working as an EPSRC Doctoral Prize Research Fellowship at the University of Sheffield, where he is working on photonic-crystal lasers.

David T. D. Childs received the B.Sc. degree in physics, the M.Sc. degree in semiconductor science and technology, and the Ph.D. degree from Imperial College, London, U.K., in 1996, 1997, and 2002, respectively. He was with the R\&D Department of Marconi Optical Components and Bookham, Caswell Semiconductor Research and Fabrication Facility, till 2006, where he was responsible for the development of a range of telecommunication lasers. Since then, he has been with the Department of Electronic and Electrical Engineering, University of Sheffield, Sheffield, U.K. He is involved in a number of projects developing semiconductor light sources from visible to terahertz wavelengths. This work spans from fundamental material improvement to advanced device engineering. He is also involved in the application of semiconductor devices to fields ranging from selective laser melting to midinfrared hyperspectral imaging. 
Pavlo Ivanov received the Engineer' degree in electronic engineering from the Kharkiv National University of Radio and Electronics, Kharkiv, Ukraine, in 1999, and the Ph.D. degree in laser optics and physics from Kharkiv National University, Kharkiv, in 2004. He was a Teaching Assistant with the Kharkiv National University of Radio and Electronics and a Visiting Fellow with the University of Bristol, Bristol, U.K., from 2003 to 2011. From 2012 to 2013, he was a Research Associate with the Leeds University, Leeds, U.K. He is currently a Research Associate at the University of Sheffield, Sheffield, U.K. He has been involved in theoretical and experimental investigation of vertical-cavity surfaceemitting lasers, incorporating 2-D photonic crystals, quantum cascade lasers, and photonic-crystal surface-emitting lasers.

Ben J. Stevens received the Ph.D. degree in advanced GaAs-based lasers from the University of Sheffield, Sheffield, U.K., in 2010. Since July 2009, he has been at the National Centre for III-V Technologies, University of Sheffield, where he is responsible for the development of MOVPE growth. His research interests include lasers and regrowth technologies. He received the Japanese Society for the Promotion of Science Summer Fellowship, which he spent in the Asakawa Group, Tsukuba, Japan, learning selective area MBE.

Nasser Babazadeh received the Ph.D. degree from the University of Sheffield, Sheffield, U.K., in 2010. Since 2010, he has been a Postdoctoral Researcher with the Department of Electronic and Electrical Engineering, University of Sheffield. His research interests include semiconductor nanostructures, with emphasis on quantum dot, quantum well structures includingVCSELS/VECSELS, and super luminescent diodes.

Jayanta Sarma received the B.Eng. degree in electronics and telecommunication engineering from Jadavpur University, Calcutta, India, the M.S. degree in electrical engineering from the Illinois Institute of Technology, Chicago, IL, USA, and the Ph.D. degree from the Department of Electronics and Electrical Engineering, University of Leeds, Leeds, U.K. He was a National Research Council of Canada Postdoctoral Research Fellow with the Communications Research Centre, Ottawa. He was a Research Associate with the University of Sheffield, the University of Liverpool, and the University of Bath. He was appointed as a Member of the Academic Staff at the University of Bath, where he led a research group working on guided-wave photonics, till he retired as a Reader.

Salam Khamas joined the University of Sheffield, Sheffield, U.K., as an Academic Staff, in 2002. He has worked in the field of antennas since 1988, when he designed the first high temperature superconducting dipole antenna. A patent was awarded and a prize winning paper was published on that work. Since then, he has worked on various aspects of antennas modeling and design, which resulted in 37 published journal papers and he has presented several papers at international conferences. His publications include 17 IEEE Transactions that propose novel modeling methods in electromagnetics. His research interests include computational electromagnetics, dielectric resonator antennas, conformal antennas, and arrays. His name was included in the 16th edition of Who's Who publication for his achievements in research.

Alex J. Crombie received the M.Phys. degree in physics from the University of Sheffield, Sheffield, U.K., in 2008, and the M.Sc. degree in electrical engineering from the University of Hull, Hull, U.K, in 2013. He is currently working toward the Ph.D. degree in photonic crystal surface emitting lasers as part of the Semiconductor Materials Group, Electrical Engineering Department.
Guangrui Li received the B.Eng. degree in electrical engineering and automation from Fudan University, Shanghai, China, in 2013, and the M.Sc. degree in semiconductor photonics from the University of Sheffield, Sheffield, U.K., in 2014, where he is currently working toward the Ph.D. degree in photoniccrystal surface-emitting lasers.

Gary Ternent received the B.Eng. degree in electrical and electronic engineering and the $\mathrm{Ph} . \mathrm{D}$. degree in developing $\mathrm{Si} / \mathrm{SiGe} \mathrm{RF}$ technology from the University of Glasgow, Glasgow, U.K., in 1993 and 2000, respectively. He was a Device Engineer with Siemens Microelectronics in North Tyneside. He was the Lead Development Engineer with Intense Ltd., where he patented a number of laser printing devices. He was the Product Manager with Xanic Ltd., developing MMICs for the defense markets. He was the Lead Engineer with the III-V Nanofabrication National Centre, University of Glasgow. He is currently a Research Fellow at the University of Glasgow, where he is responsible for the project management of laser diode integration for quantum technology in sensors and metrology. His research interests include device engineering and fabrication of nanoscale electrical and optical semiconductor devices.

Stephen Thoms was a Research Assistant till 1989, later became a Research Technologist. He has been involved in nanofabrication for approximately 30 years, since joining the University of Glasgow, Glasgow, U.K., in 1984.

He has led the operation of the Leica EBPG 5 electron beam lithography tool since its arrival in 1992, and of the Vistec VB6 tool which passed it's acceptance tests at the end of 2005. This includes maintaining a high standard of tool performance and the training of approximately 20 new RAs and Ph.D. students each year. He has also been involved in numerous fabrication projects, including the investigation of dry etch damage, with special reference to sidewall damage; the evaluation of new resists for electron beam lithography, including the development of processes for using DUV resists in e-beam; the development of electron-beam-related fabrication procedures, such as writing on nonplanar surfaces and improved alignment methods; the fabrication of phase masks for fibre optics; 30-70-nm T-gate fabrication methods using electron beam lithography for RF transistors; the development of a nanoimprint "T"-gate process, which was successfully used to fabricate HEMT devices; and III-V-based MOSFET fabrication. He is an author of more than 100 papers on nanofabrication related topics.

Haiping Zhou received the B.Sc. degree from Jilin University, Jilin, China, the M.Eng. degree from Tsinghua University, Beijing, China, both in semiconductor physics and devices, and the Ph.D. degree from the University of Glasgow, Glasgow, U.K. She is currently the Research Technologist leading the research work at the Laboratory of Plasma Processing Science and Technology, James Watt Nanofabrication Centre, University of Glasgow.

Richard A. Hogg received the Ph.D. degree in physics from the University of Sheffield, Sheffield, U.K., in 1995. He was a Postdoctoral Researcher with NTT Basic Research Laboratories, Atsugi, Japan, for two years. He was with Toshiba Research Europe's Cambridge Laboratory for three years. In 2000, he joined Agilent Technologies Fiber-Optic Component Operation, Ipswich, U.K. In 2003, he joined the Electronic and Electrical Engineering Department, University of Sheffield, where he is currently a Professor of semiconductor devices. His research group is active in developing the understanding of device physics and engineering, epitaxial processes fabrication technologies, and applications of various semiconductor laser, amplifier, and superluminescent diode devices. He received an EU-Japan Fellowship as a Visiting Researcher in Professor Arakawa's Laboratory, University of Tokyo. 